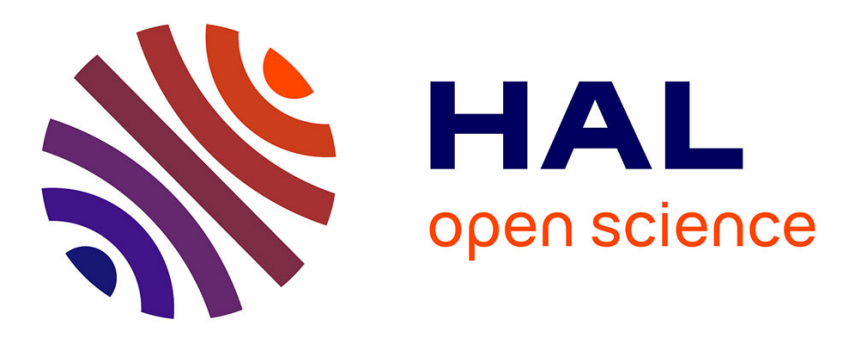

\title{
Amplification of coherent streaks in the turbulent Couette flow: an input-output analysis at low Reynolds number
}

Yongyun Hwang, Carlo Cossu

\section{- To cite this version:}

Yongyun Hwang, Carlo Cossu. Amplification of coherent streaks in the turbulent Couette flow: an input-output analysis at low Reynolds number. Journal of Fluid Mechanics, 2010, 643, pp.333-348. $10.1017 / \mathrm{s} 0022112009992151$. hal-01002542

\section{HAL Id: hal-01002542}

https://hal-polytechnique.archives-ouvertes.fr/hal-01002542

Submitted on 8 Jul 2014

HAL is a multi-disciplinary open access archive for the deposit and dissemination of scientific research documents, whether they are published or not. The documents may come from teaching and research institutions in France or abroad, or from public or private research centers.
L'archive ouverte pluridisciplinaire HAL, est destinée au dépôt et à la diffusion de documents scientifiques de niveau recherche, publiés ou non, émanant des établissements d'enseignement et de recherche français ou étrangers, des laboratoires publics ou privés. 


\title{
Amplification of coherent streaks in the turbulent Couette flow: an input-output analysis at low Reynolds number
}

\author{
YONGYUN HWANG AND CARLO COSSU † \\ Laboratorie d'Hydrodynamique (LadHyX), CNRS-École Polytechnique, F-91128 Palaiseau, France
}

(Received 6 March 2009; revised 9 September 2009; accepted 9 September 2009; first published online 5 January 2010)

We compute the optimal response of the turbulent Couette mean flow to initial conditions, harmonic and stochastic forcing at $R e=750$. The equations for the coherent perturbations are linearized near the turbulent mean flow and include the associated eddy viscosity. The mean flow is found to be linearly stable but it has the potential to amplify steamwise streaks from streamwise vortices. The most amplified structures are streamwise uniform and the largest amplifications of the energy of initial conditions and of the variance of stochastic forcing are realized by large-scale streaks having spanwise wavelengths of $4.4 h$ and $5.2 h$ respectively. These spanwise scales compare well with the ones of the coherent large-scale streaks observed in experimental realizations and direct numerical simulations of the turbulent Couette flow. The optimal response to the harmonic forcing, related to the sensitivity to boundary conditions and artificial forcing, can be very large and is obtained with steady forcing of structures with larger spanwise wavelength $(7.7 h)$. The optimal large-scale streaks are furthermore found proportional to the mean turbulent profile in the viscous sublayer and up to the buffer layer.

Key words: large-scale structures, optimal perturbations, streaks, turbulent flows

\section{Introduction}

Streamwise streaks, i.e. narrow regions where the streamwise velocity is larger or smaller than the average, are ubiquitous in wall-bounded turbulent flows. Kline et al. (1967) observed them in the near-wall region of turbulent boundary layers where they have a characteristic spanwise spacing of about one hundred wall units (Moin \& Kim 1982; Smith \& Metzler 1983). These streaks are now understood as a part of selfsustained processes playing a key role in the production of near-wall turbulence and skin friction (Jang, Benney \& Gran 1986; Jiménez \& Moin 1991; Hamilton, Kim \& Waleffe 1995; Waleffe 1995; Schoppa \& Hussain 2002). Further investigations have revealed the existence of coherent large-scale streaks extending in the outer layer and scaling with outer units. The turbulent Couette flow is one of the first cases where these large-scale streaks have been observed. Lee \& Kim (1991) found large-scale quasi-steady coherent streaks in the direct numerical simulation (DNS) of the fully 
developed turbulent Couette flow at $R e=3000$ (where $R e$ is based on the channel half-width $h$ and half of the velocity difference between the walls). These streaks, of spanwise wavelength $\lambda_{z} \approx 4 h$, occupied half of the spanwise size $\left(L_{z}=8 \pi h / 3\right)$ of the computational domain and its whole streamwise extension $\left(L_{x}=4 \pi h\right)$. The existence of these large-scale streaks was then confirmed by the experiments of Tillmark \& Alfredsson (1994). In order to understand whether the characteristics of these largescale structures were affected by the size of the computational box, Komminaho, Lundbladh \& Johansson (1996) repeated the computations in a large domain ( $L_{x}=28 \pi / h$ and $\left.L_{z}=8 \pi h\right)$ at $R e=750$. They found large-scale coherent streaks with the same spanwise wavelength $(\approx 4 h)$ of those of Lee \& Kim (1991) but more unsteady and extending more than $30 \mathrm{~h}$ in the streamwise direction. They also found that resolving these large-scale structures is essential to obtain accurate turbulent statistics. Kitoh, Nakabayashi \& Nishimura (2005), Tsukahara, Kawamura \& Shingai (2006), Tsukahara, Iwamoto \& Kawamura (2007) and Kitoh \& Umeki (2008) also found large-scale streaky structures at higher Reynolds numbers with typical size $\lambda_{z} \approx 4.2 h-5 h$. Large-scale coherent streaks have also been observed in the turbulent pipe (Kim \& Adrian 1999), in the turbulent plane Poiseuille flow (del Álamo \& Jiménez 2003) and in the turbulent boundary layer (Tomkins \& Adrian 2003, 2005; Hutchins \& Marusic 2007a). At high Reynolds numbers, these large-scale structures dominate the streamwise turbulent kinetic energy throughout the logarithmic region (Tomkins \& Adrian 2003, 2005), and modulate the cycles of near-wall structures (Hutchins \& Marusic 2007b).

In free shear layers, the appearance of large-scale coherent structures has been related to the linear inflectional instability of the turbulent mean profile (Ho \& Huerre 1984). This explanation is, however, not applicable to the large-scale coherent streaks observed in wall-bounded turbulent shear flows, such as the Couette, Poiseuille and boundary layer flows, because the mean velocity profiles of these flows are all linearly stable. For all these flows, the related mechanism observed in the laminar case is the 'lift-up' effect (Moffatt 1967; Ellingsen \& Palm 1975; Landahl 1980, 1990), by which low-energy streamwise vortices are converted into high-energy streaks in the presence of shear. The growth of the streaks by lift-up is algebraic and unbounded in inviscid flows, but is only transient in viscous flows (Gustavsson 1991). The streaks transient growth is related to the non-normality of the Navier-Stokes operator and can be maximized by using optimal vortices as initial conditions (see e.g. Trefethen et al. 1993; Farrell \& Ioannou 1996; Schmid \& Henningson 2001). Streaks of large enough amplitude undergo inflectional instabilities (Waleffe 1995; Reddy et al. 1998; Andersson et al. 2001) playing a crucial role in subcritical transition to turbulence. The optimal perturbations leading to the largest growth of streaks have been computed for virtually all canonical laminar shear flows such as the Couette, Poiseuille or Blasius solutions (Butler \& Farrell 1992; Reddy \& Henningson 1993; Trefethen et al. 1993; Schmid \& Henningson 1994).

Butler \& Farrell (1993) transposed the computation of optimal perturbations to turbulent flows using the turbulent mean flow profile as base flow and the molecular viscosity in the equations for the perturbations. For the turbulent Poiseuille flow, they found an optimal spanwise wavelength $\lambda_{z}=3 h$ close to the one found in the laminar case. They were able to retrieve the optimal spanwise wavelength $\lambda_{z}^{+} \approx 100$ typical of the near-wall streaks by constraining the optimization time to an appropriate value. The use of the molecular viscosity and the subsequent necessary enforcement of characteristic time scales also appear in the investigations of the response to stochastic forcing by Farrell \& Ioannou $(1993 a, 1998)$. Later, del Álamo \& Jiménez 
(2006) and Pujals et al. (2009) have recomputed the optimal growths sustained by the turbulent Poiseuille flow including an effective turbulent viscosity in the linearized equation according to the approach used by Reynolds \& Hussain (1972). Without any additional constraint on the optimization time, they find that at sufficiently high Reynolds numbers two locally optimal spanwise wavelengths exist respectively scaling with outer and inner units. The maximum growth was obtained for $\lambda_{z}=4 h$, in good agreement with the observations of large-scale streaks. The secondary maximum of the growth was found to scale in inner units and it was reached for $\lambda_{z}^{+}=92$, which corresponds well to the spanwise spacing of the near-wall streaks. These findings suggest that a strong relation exists between the large-scale streaks patterns selected by the optimal transient energy growth and those observed in turbulent wall-bounded shear flows. Considering turbulent boundary layer profiles, Cossu, Pujals \& Depardon (2009) have also found the two peaks of energy amplification respectively related to near-wall and large-scale streaks. However, in that case the spanwise wavelength optimizing the energy growth of large-scale streaks was found to be larger than the one of the large-scale coherent streaks actually observed.

No investigation of the optimal energy amplifications sustained by the turbulent Couette flow is currently available, even if the presence of large-scale coherent streaks with a spanwise spacing of $4 h-5 h$ is a well-established feature of this flow. Furthermore, del Álamo \& Jiménez (2006), Cossu et al. (2009) and Pujals et al. (2009) have only considered the optimal temporal energy growth whereas the optimal response to harmonic and stochastic forcing may be equally relevant and their computation is now routine for the canonical laminar flows (Farrell \& Ioannou 1993a,b; Bamieh \& Dahleh 2001; Jovanović \& Bamieh 2005; Fontane, Brancher \& Fabre 2008). In this respect, some relevant questions are still unanswered: What are the streamwise and spanwise scales most amplified by the turbulent Couette flow in the initial value and in the harmonic and stochastic forcing problems? How do they compare between them and with analogous results found in laminar flows? What is the shape of the corresponding optimal perturbations? How do these structures relate to the large-scale coherent structures observed in experiments and DNS? Do their spanwise scales relate well to the most energetic ones, as found in the turbulent Poiseuille flow, or are they larger, as found in the turbulent boundary layer?

In order to answer these questions, we have conducted an input-output analysis of the turbulent Couette mean flow by considering its optimal response to an initial condition and to harmonic forcing, and its response to stochastic forcing. In the latter case, the structures that contribute most to the variance of the response have also been computed. The turbulent mean flow is computed by DNS. Our results have been obtained at the low Reynolds number $R e=750$ corresponding to $R e_{\tau} \approx 52$, which is a well-documented case with respect to both the large scale structures characteristics and the convergence of the mean profile with respect to the size of the computational box. Well converged, but less documented, results from DNS are currently only available for $R e_{\tau} \lesssim 200$, which is well below the regime $\left(R e_{\tau} \gtrsim 500\right.$ according to Pujals et al. 2009) where the primary and secondary peaks begin to be well separated and where well-defined asymptotic scalings in $R e_{\tau}$ can be obtained. There is therefore not great advantage in considering slightly larger Reynolds numbers.

The paper is organized as follows: In $\S 2$, we briefly introduce the mathematical problem to be solved and describe the tools used to numerically solve it. The main results are reported and compared with previous findings in $\S 3$. A summary of the main findings and a discussion of their implications are given in $\S 4$. 


\section{Background}

\subsection{Turbulent mean flow and eddy viscosity}

We consider the plane Couette flow of a viscous fluid of kinematic viscosity $v$ and constant density $\rho$ between two parallel plates located at $y= \pm h$, where we denote by $x, y$ and $z$ the streamwise, wall-normal and spanwise coordinates respectively. The plates move in opposite directions with velocity $\left( \pm U_{w}, 0,0\right)$. For sufficiently high values of the Reynolds number $R e=h U_{w} / v$, the flow is turbulent. DNS is used to compute the turbulent mean velocity profile $U(y)$ that is obtained by averaging the instantaneous fields in space (over horizontal planes in the computational box) and in time. Once that $U(y)$ is known, it is straightforward to compute the mean shear stress at the wall $\tau_{w} / \rho=\nu \mathrm{d} U /\left.\mathrm{d} y\right|_{w}$, the friction velocity $u_{\tau}=\sqrt{\tau_{w} / \rho}$ and the friction Reynolds number $R e_{\tau}=u_{\tau} h / v$. Since the mean pressure gradient is zero in the Couette flow, the mean shear stress $\tau=-\rho \overline{u^{\prime} v^{\prime}}+\rho \nu \mathrm{d} U / \mathrm{d} y$ (where $u^{\prime}$ and $v^{\prime}$ are the streamwise and the wall-normal velocity fluctuations, respectively) is constant and equal to its value at the wall $\tau_{w}$. By introducing the eddy viscosity $v_{t}(y)=-\overline{u^{\prime} v^{\prime}} /(\mathrm{d} U / \mathrm{d} y)$, it is found that

$$
\left[\frac{v_{t}(y)}{v}+1\right] \frac{\mathrm{d}\left(U / u_{\tau}\right)}{\mathrm{d}(y / h)}=R e_{\tau} .
$$

The (total) effective viscosity is then defined as $v_{T}=v_{t}+v$.

\subsection{Generalized Orr-Sommerfeld-Squire equations}

Following the approach of Reynolds \& Hussain (1972), del Álamo \& Jiménez (2006), Cossu et al. (2009) and Pujals et al. (2009), we consider the linearized equations satisfied by small coherent perturbations in the presence of the associated effective viscosity $\nu_{T}(y)$ :

$$
\begin{gathered}
\nabla \cdot \boldsymbol{u}=0, \\
\frac{\partial \boldsymbol{u}}{\partial t}+\nabla \boldsymbol{u} \cdot \boldsymbol{U}+\nabla \boldsymbol{U} \cdot \boldsymbol{u}=-\frac{1}{\rho} \nabla p+\nabla \cdot\left[v_{T}\left(\nabla \boldsymbol{u}+\nabla \boldsymbol{u}^{T}\right)\right]+\boldsymbol{f},
\end{gathered}
$$

where $\boldsymbol{U}=(U, 0,0)$ and $\boldsymbol{u}=(u, v, w)$ are the mean and the perturbation velocities respectively, $p$ is the perturbation pressure and $\boldsymbol{f}=\left(f_{u}, f_{v}, f_{w}\right)$ is a forcing term. The streamwise and spanwise homogeneity of the problem allow to consider separately each in-plane Fourier mode $\hat{\boldsymbol{u}}(y, t ; \alpha, \beta) \mathrm{e}^{\mathrm{i}(\alpha x+\beta z)}$ and $\hat{\boldsymbol{f}}(y, t ; \alpha, \beta) \mathrm{e}^{\mathrm{i}(\alpha x+\beta z)}$, where $\alpha$ and $\beta$ are the streamwise and spanwise wavenumbers respectively. The following generalized Orr-Sommerfeld-Squire system is obtained from (2.2):

$$
\begin{aligned}
\frac{\partial}{\partial t}\left[\begin{array}{l}
\hat{v} \\
\hat{\eta}
\end{array}\right]= & \underbrace{\left[\begin{array}{cc}
\Delta^{-1} \mathscr{L}_{\mathscr{O S}} & 0 \\
-\mathrm{i} \beta U^{\prime} & \mathscr{L}_{\mathscr{S} Q}
\end{array}\right]}_{\boldsymbol{A}}\left[\begin{array}{l}
\hat{v} \\
\hat{\eta}
\end{array}\right] \\
& +\underbrace{\left[\begin{array}{ccc}
-\mathrm{i} \alpha \Delta^{-1} \mathscr{D} & -k^{2} \Delta^{-1} & -\mathrm{i} \beta \Delta^{-1} \mathscr{D} \\
\mathrm{i} \beta & 0 & -\mathrm{i} \alpha
\end{array}\right]}_{\boldsymbol{B}}\left[\begin{array}{c}
\hat{f}_{u} \\
\hat{f}_{v} \\
\hat{f}_{w}
\end{array}\right],
\end{aligned}
$$

with the generalized Orr-Sommerfeld-Squire operators, given by (e.g. Reynolds \& Hussain 1972; Cossu et al. 2009; Pujals et al. 2009):

$$
\begin{aligned}
& \mathscr{L}_{\mathscr{O S}}=-\mathrm{i} \alpha\left(U \Delta-U^{\prime \prime}\right)+v_{T} \Delta^{2}+2 v_{T}^{\prime} \Delta \mathscr{D}+v_{T}^{\prime \prime}\left(\mathscr{D}^{2}+k^{2}\right), \\
& \mathscr{L}_{\mathscr{Y} \mathscr{Q}}=-\mathrm{i} \alpha U+v_{T} \Delta+v_{T}^{\prime} \mathscr{D} .
\end{aligned}
$$


Here, $\mathscr{D}$ and ' denote $\partial / \partial y, \Delta=\mathscr{D}^{2}-k^{2}, k^{2}=\alpha^{2}+\beta^{2}$ and $\hat{\eta}$ is the wall-normal vorticity Fourier mode. The system is completed by the initial condition $\left.\hat{\boldsymbol{u}}\right|_{t=0}=\hat{\boldsymbol{u}}_{0}$ and homogeneous boundary conditions for the velocity perturbations on the walls, which result in $\hat{v}(y= \pm h)=0, \mathscr{D} \hat{v}( \pm h)=0$ and $\hat{\eta}( \pm h)=0$. The velocity components can be retrieved from the wall-normal variables with

$$
\left[\begin{array}{c}
\hat{u} \\
\hat{v} \\
\hat{w}
\end{array}\right]=\underbrace{\frac{1}{k^{2}}\left[\begin{array}{cc}
\mathrm{i} \alpha \mathscr{D} & -\mathrm{i} \beta \\
k^{2} & 0 \\
\mathrm{i} \beta \mathscr{D} & \mathrm{i} \alpha
\end{array}\right]}_{C}\left[\begin{array}{l}
\hat{v} \\
\hat{\eta}
\end{array}\right] .
$$

\subsection{Optimal perturbations}

We consider the optimal response of the system (2.3) to initial conditions, harmonic forcing and stochastic forcing. The definition of these optimals, briefly recalled below, is quite standard and can be found, for example, in the monographs by Farrell \& Ioannou (1996) and Schmid \& Henningson (2001). The optimal temporal energy growth of $(\alpha, \beta)$ modes is found by optimizing over the shape of the initial condition the ratio of the energy of the response at a given time $t$ to the energy of the initial condition:

$$
G(t ; \alpha, \beta) \equiv \max _{\hat{\boldsymbol{u}}_{0} \neq \mathbf{0}} \frac{\|\hat{\boldsymbol{u}}(t ; \alpha, \beta)\|^{2}}{\left\|\hat{\boldsymbol{u}}_{0}(\alpha, \beta)\right\|^{2}},
$$

where $\|\hat{\boldsymbol{u}}\|^{2}=\int_{-h}^{h} \hat{\boldsymbol{u}}^{H} \hat{\boldsymbol{u}} \mathrm{d} y=\int_{-h}^{h}|\hat{\boldsymbol{u}}|^{2}+|\hat{\boldsymbol{v}}|^{2}+|\hat{\boldsymbol{w}}|^{2} \mathrm{~d} y$. The maximum growth is defined by further maximizing in time $G_{\max }(\alpha, \beta) \equiv \max _{t} G(t ; \alpha, \beta)$ and it is attained at $t=t_{\max }$.

When harmonic forcing $\hat{\boldsymbol{f}}(y, t)=\tilde{\boldsymbol{f}}(y) \mathrm{e}^{-\mathrm{i} \omega_{f} t}$ is applied with frequency $\omega_{f}$, the response $\hat{\boldsymbol{u}}(y, t)=\tilde{\boldsymbol{u}}(y) \mathrm{e}^{-\mathrm{i} \omega_{f} t}$ is observed after the switch-on transients have decayed, assuming that the system is stable. In this case, the optimal response is the one having the maximum ratio of the energy of the response to the energy of the forcing:

$$
R\left(\omega_{f} ; \alpha, \beta\right)=\max _{\tilde{\boldsymbol{f}} \neq \mathbf{0}} \frac{\left\|\tilde{\boldsymbol{u}}\left(\omega_{f} ; \alpha, \beta\right)\right\|^{2}}{\left\|\tilde{\boldsymbol{f}}\left(\omega_{f} ; \alpha, \beta\right)\right\|^{2}} .
$$

The optimal response $R\left(\omega_{f}\right)$ is given by the norm of the resolvent operator along the imaginary axis $\zeta=-\mathrm{i} \omega_{f}$ (e.g. Trefethen et al. 1993). The maximum response $R_{\max }(\alpha, \beta)=\max _{\omega_{f}} R\left(\omega_{f} ; \alpha, \beta\right)$ is obtained with $\omega_{f, \max }$, and is also referred to as $H_{\infty}$ norm of the transfer function relating the forcing to the response (Zhou, Doyle \& Glover 1996).

The response to the stochastic forcing $\hat{\boldsymbol{f}}(t, y) \mathrm{e}^{\mathrm{i}(\alpha x+\beta z)}$ with Gaussian probability distribution and zero mean value $\langle\hat{\boldsymbol{f}}\rangle=\mathbf{0}$ is finally considered. The forcing is assumed to be delta-correlated in time: $\left\langle\hat{\boldsymbol{f}}(y, t) \otimes \hat{\boldsymbol{f}}^{*}\left(y^{\prime}, t^{\prime}\right)\right\rangle=\boldsymbol{R} \delta\left(t-t^{\prime}\right)$ where * denotes complex conjugation and $\delta(t)$ is the Dirac's delta function. We also assume, without loss of generality, that $\boldsymbol{R}=\boldsymbol{I}$. The variance of the response, $V(\alpha, \beta)=\left\langle\|\hat{\boldsymbol{u}}\|^{2}\right\rangle$, also referred to as $\mathrm{H}_{2}$ norm of the transfer function relating the forcing to the output (Zhou et al. 1996), is given by

$$
V(\alpha, \beta)=\operatorname{trace}\left(\boldsymbol{C} \boldsymbol{X}_{\infty} \boldsymbol{C}^{\dagger}\right),
$$

where the superscript ${ }^{\dagger}$ denotes the adjoint with respect to the standard inner product (after discretization $\boldsymbol{C}^{\dagger}$ would simply be the Hermitian of $\boldsymbol{C}$ ). $\boldsymbol{X}_{\infty}$ is the solution of the following algebraic Lyapunov equation:

$$
\boldsymbol{A} \boldsymbol{X}_{\infty}+\boldsymbol{X}_{\infty} \boldsymbol{A}^{\dagger}+\boldsymbol{B} \boldsymbol{B}^{\dagger}=\mathbf{0} .
$$


Since $\boldsymbol{C} \boldsymbol{X}_{\infty} \boldsymbol{C}^{\dagger}$ is a self-adjoint operator, it has real eigenvalues $\sigma_{j}$ and a set of mutually orthogonal eigenfunctions that are usually referred to as "empirical orthogonal functions' (EOF) or Karhunen-Loève (KL) or 'proper orthogonal decomposition' (POD) modes. The variance being $V=\sum \sigma_{j}$, the ratio $\sigma_{j} / V$ represents the contribution of the $j$ th mode to the (total) variance. The mode corresponding to the largest $\sigma_{j}$ is the optimal one in the sense that it contributes most to the total variance of the system. The part of the stochastic forcing accounting for that optimal mode is computed by solving the dual Lyapunov problem. For further details, the interested reader is referred to the papers by Farrell \& Ioannou (1993a,b), Zhou et al. (1996), Bamieh \& Dahleh (2001) and Jovanovic \& Bamieh (2005) and Schmid (2007).

\subsection{Numerical tools}

The DNS of the turbulent channel flow, necessary to compute the mean flow, has been performed using the channelflow code (Gibson, Halcrow \& Cvitanovic 2008) that integrates the incompressible Navier-Stokes equation using a spectral method based on the Fourier-Galerkin discretization in the streamwise and spanwise directions, and the Chebyshev-tau discretization in the wall-normal direction. The solution is advanced in time using a third-order semi-implicit time-stepping. Dealiasing with the $2 / 3$ rule is implemented in the streamwise and spanwise directions.

The generalized Orr-Sommerfeld-Squire system (2.3) is discretized using a Chebyshev-collocation method with $N_{y}$ collocation points in the wall-normal direction. The differentiation operators are discretized using the Chebyshev differentiation matrices of Weideman \& Reddy (2000) that include the appropriate boundary conditions for $\hat{v}$ and $\hat{\eta}$. The optimal transient growth and the optimal harmonic response are computed using standard methods described, for example, in Schmid \& Henningson (2001) which are implemented in the well validated code used in the channel and boundary layer computations of Pujals et al. (2009) and Cossu et al. (2009). The Lyapunov equation (2.8), discretized by the same method, was solved using the lyap function in matlab. The algorithms used to compute the optimal harmonic and stochastic responses have been validated by comparing the results obtained for laminar Couette and Poiseulle flows with those of Reddy \& Henningson (1993), Schmid \& Henningson (2001) and Jovanović \& Bamieh (2005). The results in the present study are obtained with $N_{y}=257$. We have verified, for some selected case, that the results do not change in any appreciable way if the number of collocation points is doubled to $N_{y}=513$.

\section{Results}

\subsection{Turbulent mean flow and the Reynolds stress}

The turbulent mean flow and the associated Reynolds shear stress computed by DNS at $R e=750$ are reported in figure 1 . To obtain well-converged results we have used the same computational box $\left(L_{x} \times L_{y} \times L_{z}=28 \pi h \times 2 h \times 8 \pi h\right)$ and resolution $\left(N_{x} \times N_{y} \times N_{z}=340 \times 55 \times 170\right.$ after dealiasing $)$ used by Komminaho et al. (1996). The turbulent flow is allowed to develop up to $t=800 h / U_{w}$ and then the mean quantities are obtained by averaging in the symmetric horizontal planes $\pm y$ and in time for $t \in[800,1500] h / U_{w}$. $R e_{\tau}$ converges to the same $R e_{\tau}=52$ found by Komminaho et al. (1996) and shows also good agreement with the experimental value $R e_{\tau}=50$ reported by Kitoh et al. (2005). The mean shear rate at the centreline $\mathrm{d}\left(U / U_{w}\right) /\left.\mathrm{d}(y / h)\right|_{y=0}=0.1865$ is also in good accordance with the 0.18 value found 

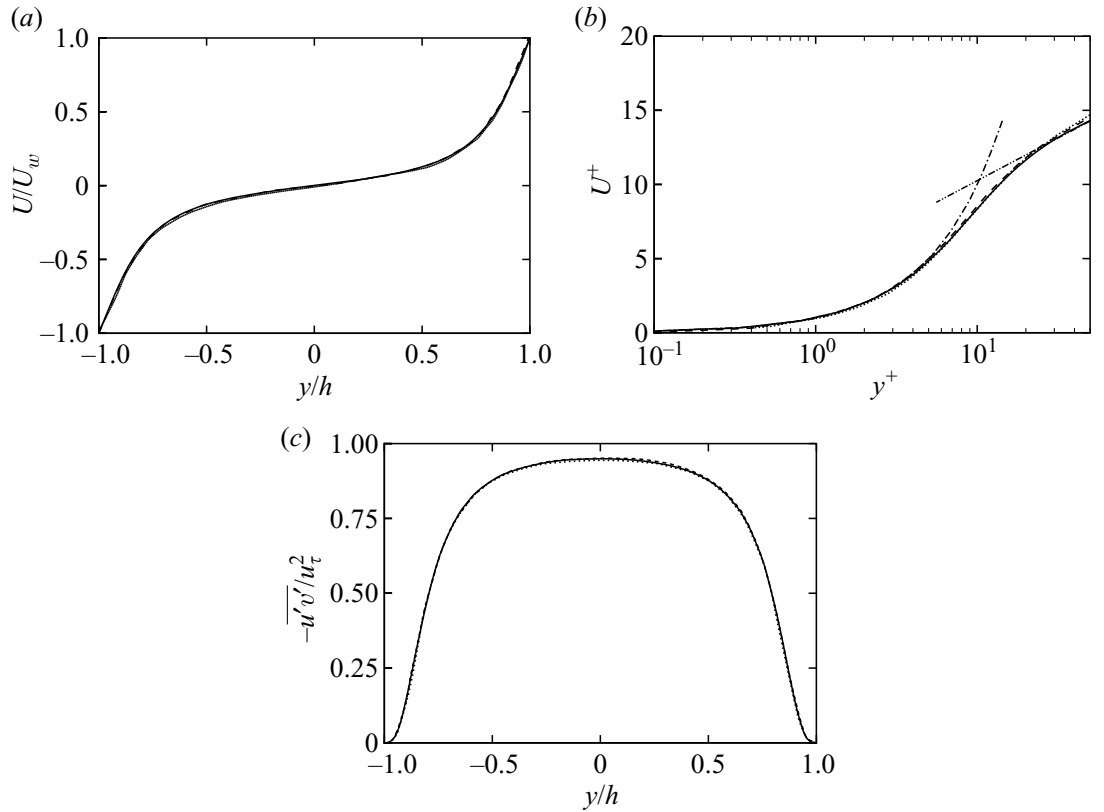

FiguRE 1. Turbulent mean velocity profile and shear stress from DNS. (a) Mean velocity profile expressed in outer units: present DNS (- ) compared with the profiles found by Komminaho et al. (1996) (- - - ) and Tsukahara et al. (2006) (….....); the curves are almost undistinguishable. (b) Same profile from the DNS (- ${ }^{-}$expressed in inner units $U^{+} \equiv\left(U+U_{w}\right) / u_{\tau}$ and $y^{+}=(y+h) u_{\tau} / v$ and compared to the curves $U^{+}=y^{+}(-\cdot-\cdot-)$ and $U^{+}=(1 / 0.4) \log y^{+}+4.5(-\cdots-\cdots-)$. (c) Turbulent mean shear stress $-\overline{u^{\prime} v^{\prime}} / u_{\tau}^{2}$ from the present DNS (- ) and from the data of Komminaho et al. (1996) (- - - ) and Tsukahara et al. (2006) $(\cdots \cdots \cdots)$; the curves are almost undistinguishable.

by Komminaho et al. (1996) and the experimental value of 0.2 (Tillmark 1995; Kitoh et al. 2005). As shown in figure 1, the computed mean flow and the associated Reynolds shear stress are almost undistinguishable from from the ones computed by Komminaho et al. (1996) and by Tsukahara et al. (2006).

\subsection{Optimal response to initial conditions, harmonic and stochastic forcing}

As a preliminary step, the eigenvalues of the Orr-Sommerfeld-Squire operator equation (2.3) have been computed and found to be stable. The optimal response to initial condition, harmonic and stochastic forcing have then been computed for a set of wavenumbers $\alpha$ and $\beta$ ranging from 0 to $5 / h$ and from 0 to $20 / h$ respectively. The optimal temporal energy growths $G(t, \alpha, \beta)$ are computed up to $t=100 h / U_{w}$ with the resolution $\Delta t=0.05 h / U_{w}$ allowing the extraction of the respective $G_{\max }(\alpha, \beta)$ reported in figure 2. Only elongated structures, roughly the ones with $\alpha<\beta$, are significantly amplified, the most amplified ones being streamwise uniform $(\alpha=0$ i.e. $\left.\lambda_{x}=\infty\right)$. The energy growths $G_{\max }$ are not very large, attaining a maximum value of 6.49. For streamwise uniform perturbations, the most amplified spanwise wavenumber is $\beta=1.43 / h$ corresponding to the spanwise wavelength $\lambda_{z}=4.4 h$. As $\alpha$ increases, the most amplified $\beta$ slightly increases: e.g. $\beta=1.46 / h\left(\lambda_{z}=4.3 h\right)$ for $\alpha=0.1 / h\left(\lambda_{x}=63 h\right), \beta=1.57 / h\left(\lambda_{z}=4.0 h\right)$ for $\alpha=0.25 / h\left(\lambda_{x}=25 h\right)$ and $\beta=1.75 / h$ $\left(\lambda_{z}=3.6 h\right)$ for $\alpha=0.5 / h\left(\lambda_{x}=12.5 h\right)$. 


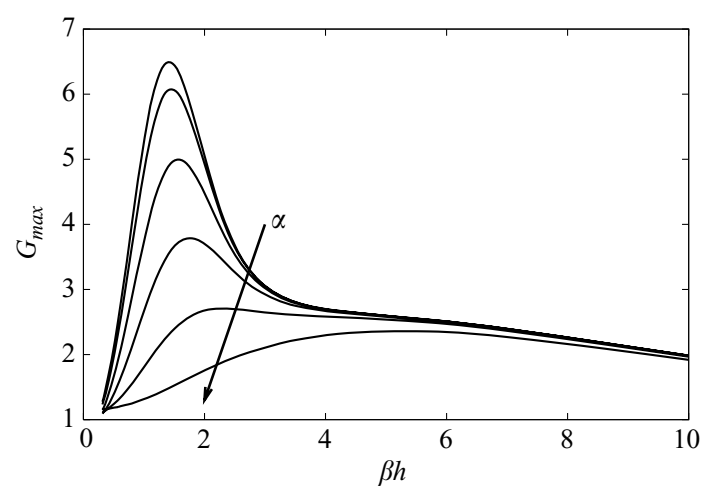

FIGURE 2. Dependence of the maximum temporal energy growth $G_{\max }$ on the dimensionless spanwise wavenumber $\beta h$ for selected streamwise wavenumbers (top to bottom: $\alpha h=$ $0,0.1,0.25,0.5,1,2)$.
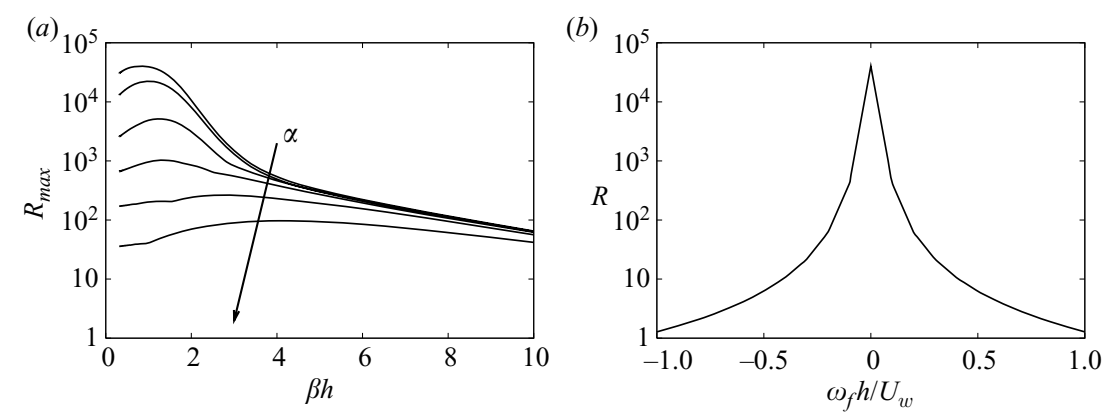

FIGURE 3. (a) Dependence of the maximum energy amplification of harmonic forcing $R_{\max }$ on the dimensionless spanwise wavenumber $\beta h$ for selected streamwise wavenumbers $\alpha$ (top to bottom: $\alpha h=0,0.1,0.25,0.5,1,2)$. (b) Dependence of the optimal energy amplification $R$ on the dimensionless harmonic forcing frequency $\omega_{f} h / U_{w}$ for the optimal wavenumbers $(\alpha=0$, $\beta h=0.82)$.

The optimal response to harmonic forcing $R\left(\omega_{f}, \alpha, \beta\right)$ is computed for the same set of wavenumbers. A set of forcing frequencies $\omega_{f}$ ranging from $-10 U_{w} / h$ to $10 U_{w} / h$ with resolution $\Delta \omega_{f}=0.1 U_{w} / h$ is considered, and the maximum response $R_{\max }(\alpha, \beta)$ extracted from these data is reported in figure 3(a). Similarly to the optimal transient growth, only elongated structures are appreciably amplified. For streamwise uniform structures, the largest response $\left(R_{\max }=40269\right)$ is obtained for $\beta=0.82 / h$ (corresponding to $\lambda_{z}=7.7 h$ ). The dependence of the optimal response on the forcing frequency is shown in figure $3(a)$ for the most amplified wavenumbers $(\alpha=0, \beta=0.82 / h)$. From this figure it is seen that the largest response is obtained when the forcing is steady $\left(\omega_{f, \max }=0\right)$ and that the frequency response is strongly concentrated near $\omega_{f}=0$. This indicates that the system behaves like a strongly selective low-pass frequency filter.

Finally, the variance $V(\alpha, \beta)$ of the response maintained by the stochastic forcing is computed, always for the same set of wavenumbers and it is shown in figure $4(a)$. The selection of elongated structures appears also in this case. The maximum amplification of the variance $(V=377)$ is obtained with streamwise uniform structures and the spanwise wavenumber $\beta=1.21 / h$ (corresponding to $\lambda_{z \max }=5.2 h$ ). The structures with the largest contribution to the variance are then identified using the 


\begin{tabular}{lcccc} 
& \multicolumn{3}{c}{ Optimal $\lambda_{z} / h$} & \\
\cline { 2 - 4 } Flow & $G_{\max }$ & $R_{\max }$ & $V$ & $R e$ \\
Turbulent Couette & $4.4^{(*)}$ & $7.7^{(*)}$ & $5.2^{(*)}$ & 750 \\
Laminar Couette & $3.9^{(a)}$ & $5.3^{(a)}$ & $4.5^{(b)}$ & $R e \gg 1$ \\
Turbulent Poiseulle & $4.0^{(c)}$ & - & - & $R e \gg 1$ \\
Laminar Poiseulle & $3.1^{(a)}$ & $3.9^{(a)}$ & $3.5^{(b)}$ & $R e \gg 1$
\end{tabular}

TABLE 1. Optimal spanwise wavenumbers of the maximum responses to initial perturbation, harmonic forcing, and stochastic excitation. Results from: $(*)$ the present investigation, (a) Trefethen et al. (1993), (b) Jovanović \& Bamieh (2005) and (c) Pujals et al. (2009).
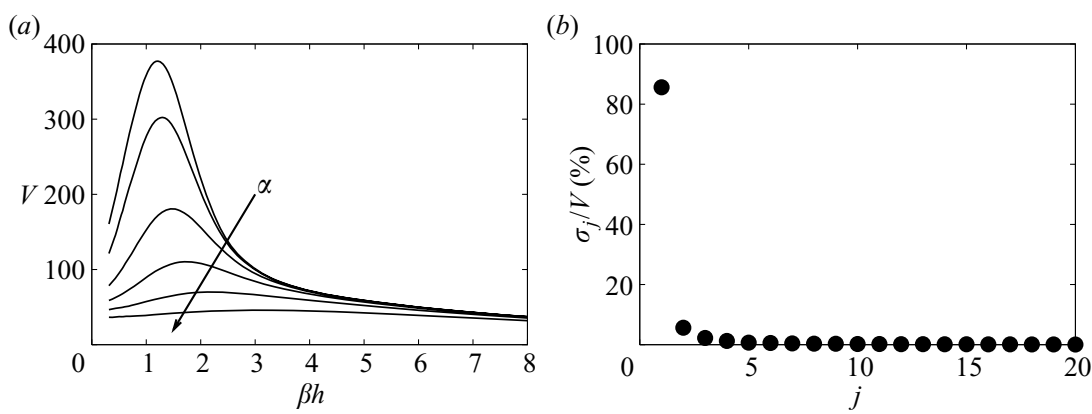

FiguRE 4. (a) Dependence of the variance $V$ of the response to stochastic forcing on the dimensionless spanwise wavenumber $\beta h$ for selected streamwise wavenumbers $\alpha$ (same values as in figures 2 and 3: top to bottom $\alpha h=0,0.1,0.25,0.5,1,2)$. (b) Contribution of the leading 20 Karhunen-Loève modes to the total variance.

Karhunen-Loève decomposition. The twenty largest ratios $\sigma_{j} / V$, representing the contribution of the $j$ th mode to the total variance, are reported in figure $4(b)$ for the wavenumbers associated to the largest variance $(\alpha=0, \beta=1.21 / h)$. We find that the most energetic mode contributes to $85 \%$ of the total maintained energy variance, implying that a unique coherent structure strongly dominates the stochastic response in this case.

\subsection{Comparison with previous results}

The fact that for all the three types of response, the maximum energy amplification is obtained by streamwise uniform structures $(\alpha=0)$ is in accordance with the similar analyses of other turbulent and laminar flows except possibly only the maximum temporal growth of the laminar Couette flow that is obtained with perturbations non-streamwise uniform, but having a very large streamwise wavelength $\left(\lambda_{x}=180 h\right)$. The most amplified spanwise wavelength is not the same for the different types of problem: The largest $\left(\lambda_{z}=7.7 h\right)$ is found for the harmonic forcing whereas the shortest $\left(\lambda_{z}=4.4 h\right)$ is found for the initial value problem, the stochastic forcing one being located in between $\left(\lambda_{z}=5.2 h\right)$. The same ordering of spanwise optimal wavelengths is observed for the laminar Couette and Poiseuille flows (see table 1). Moreover, the spanwise wavelength maximizing $G_{\max }$ in the turbulent Couette case is slightly larger than the one found for the laminar case. This is also in accordance with what is observed, for example, for the turbulent Poiseuille flow (as reported in table 1).

At the very low Reynolds number considered here $\left(R e_{\tau}=52\right)$, the small temporal energy growths that we find $\left(G_{\max } \leqslant 6.5\right)$ are not surprising if compared, for example, 


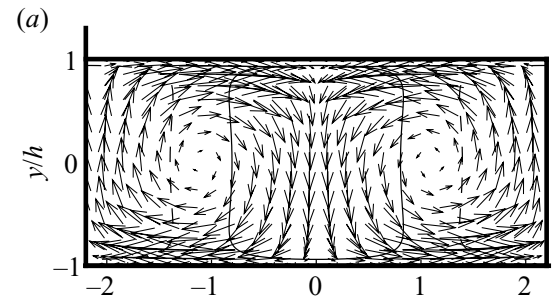

(b)
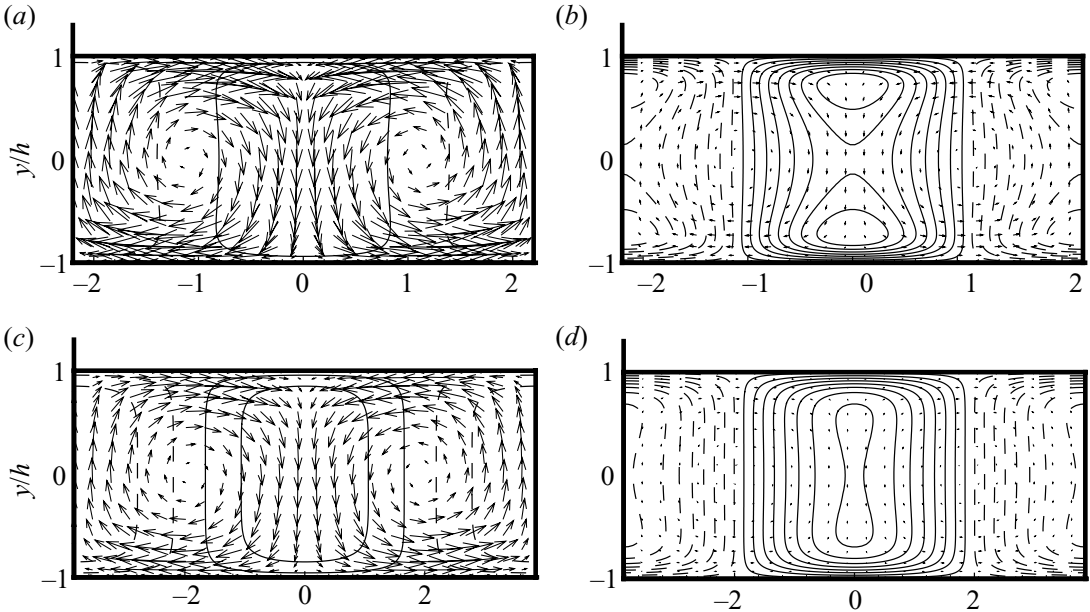

(d)
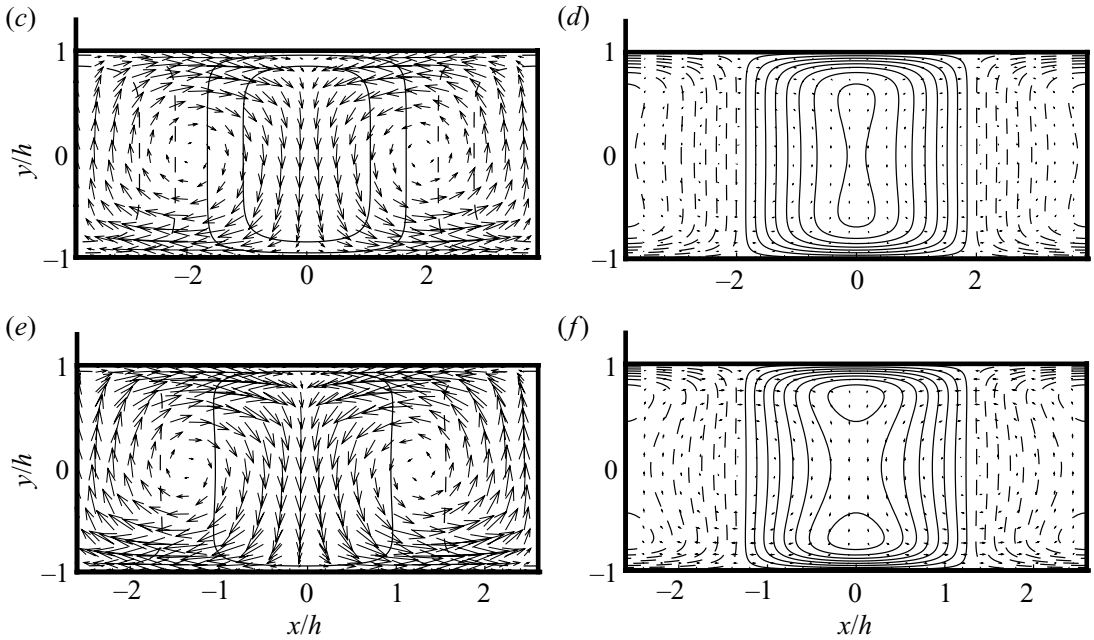

Figure 5. Cross-stream ( $y-z$ plane) view of the optimal input $(a, c, e)$ and output $(b, d, f)$ perturbation fields of the initial value problem $(a, b)$, the response to harmonic $(c, d)$ and stochastic $(e, f)$ forcing problems. Each field corresponds to the respective optimal wavenumbers $\alpha=0$ and $\beta h=1.43\left(\lambda_{z}=4.4 h\right)$ for the initial value problem $\beta h=0.82\left(\lambda_{z}=7.7 h\right)$ for the response to harmonic forcing, and $\beta h=1.21\left(\lambda_{z}=5.2 h\right)$ for the response to stochastic forcing. In all the plots the solid and dashed contours denote respectively positive and negative values of the streamwise component (with increment of 0.1 the maximum value of the output) while the cross-stream components are represented as vectors (same scales in input and output plots).

to the turbulent Poiseulle flow case where a maximum growth of the order of 10 is observed for $R e_{\tau}=500$ (Pujals et al. 2009). The low Reynolds number also explains that the $G_{\max }$ curves do not show any sign of the secondary peak associated with near-wall structures with $\lambda_{z}^{+} \approx 90-100$, contrary to what is found by del Álamo \& Jiménez (2006) and Pujals et al. (2009) for the plane Poiseuille flow. In these studies, the secondary peak appeared separated from the primary peak only for sufficiently large Reynolds numbers (typically $R e_{\tau}$ larger than $\approx 500$ ), i.e. when the inner and outer scales are sufficiently separated. The two scales are not separated in the present case $\left(R e_{\tau}=52\right)$ where the expected inner peak value $\lambda_{z}^{+} \approx 100$ corresponds to $\lambda_{z} \approx 2 h$, which is well in the range of the primary peak.

\subsection{Spatial structure of the optimal input and output perturbations}

The optimal inputs and outputs respectively associated to the maximum energy amplification in time and to the maximum response to harmonic and stochastic forcing are shown in figure 5. The optimals displayed are all uniform in the streamwise direction but their spanwise wavelength is not the same. The physical meaning of each input or output is also different. For the case of the optimal temporal energy 
growth the input (figure $5 a$ ) is the optimal initial condition (it is a velocity field) given at $t=0$ and the optimal output (figure $5 b$ ) is the velocity field of maximum energy obtained at $t_{\max }$ (here $t_{\max }=22.8 \mathrm{~h} / U_{w}$ ). For the harmonic forcing problem, the input (figure $5 c$ ) is the optimal harmonic forcing term $f$ while the output (figure $5 d$ ) is the velocity field of the corresponding harmonic response at the same frequency in the permanent regime. As here the optimal frequency is $\omega_{f}=0$, only a plot of the input and of the output is needed, as they do not change in time. Finally, for the stochastic forcing problem, the optimal input (figure $5 e$ ) and output (figure $5 f$ ) are respectively the forcing term and the associated response corresponding to the most energetic Karhunen-Loève mode representing $85 \%$ of total variance of the stochastically forced system. What is however similar in these three different cases is that for all the considered outputs most of the energy lies in the streamwise velocity component while for all the input fields most of the energy is in the cross-stream components. This implies that the dominant physical mechanism at work in the three cases is the lift-up effect by which streamwise vortices efficiently induce streamwise streaks. This is also in accordance with previous results such as, e.g. the ones of Gustavsson (1991) and Butler \& Farrell (1992) for the optimal temporal response, Trefethen et al. (1993) and Reddy \& Henningson (1993) for the optimal harmonic response (including complex frequencies) and Farrell \& Ioannou (1993b) and Jovanović \& Bamieh (2005) for the optimal stochastic response of laminar channel flows (see also e.g. Schmid \& Henningson 2001; Schmid 2007, for a review), and the results of del Álamo \& Jiménez (2006) and Pujals et al. (2009) for the optimal temporal response of the turbulent Poiseuille flow.

The wall-normal Fourier components $\left(\hat{v}\right.$ and $\left.\hat{f}_{v}\right)$ of the optimal inputs and the streamwise Fourier component $(\hat{u})$ of the optimal outputs shown in figure 5 are reported in figures $6(a)$ and $6(b)$ respectively. The wall-normal components of the optimal inputs have almost the same shape (figure $6 a$ ). The streak shapes of the corresponding optimal outputs show two maxima at $y / h= \pm 0.72$ for the optimal temporal response, $y / h= \pm 0.68$ for the leading KL mode while the streaks corresponding to the (deterministic) harmonic forcing are almost uniform in the bulk region $(|y / h| \lesssim 0.5)$.

In the case of the turbulent Poiseuille and boundary layer flows, Pujals et al. (2009) and Cossu et al. (2009) have found that the optimal streaks (output) of the initial value problem are proportional to the mean velocity profile $U^{+}\left(y^{+}\right)$in the near-wall region up to the overlap region $\left(y^{+} \lesssim 100\right)$ for large Reynolds numbers (typically larger than $R e_{\tau}=500$ for the turbulent Poiseuille flow). In the present study, this extension to the outer layer cannot be observed due to the low Reynolds number considered here $R e_{\tau}=52$ (the channel centreline corresponds to $y^{+}=52$ ). We anyway replot in inner units (in figure $6 c$ ) the profile of the optimal streaks of the temporal problem but also those of the harmonic and stochastic forcing problems already reported in figure $6(b)$. All the optimal output streaks profiles are proportional to the mean velocity profile $U$ up to the buffer layer $\left(y^{+} \lesssim 8-10\right)$. This partially extends to the optimal large-scale streaks of the forced problems the similitude observed for optimal large-scale streaks of the initial value problem.

\section{Summary and discussion}

In the present study, we have computed the optimal energy amplifications of coherent structures sustained by the turbulent Couette mean flow at $R e=750$. The turbulent mean flow has been obtained by DNS and the dynamics of the coherent 

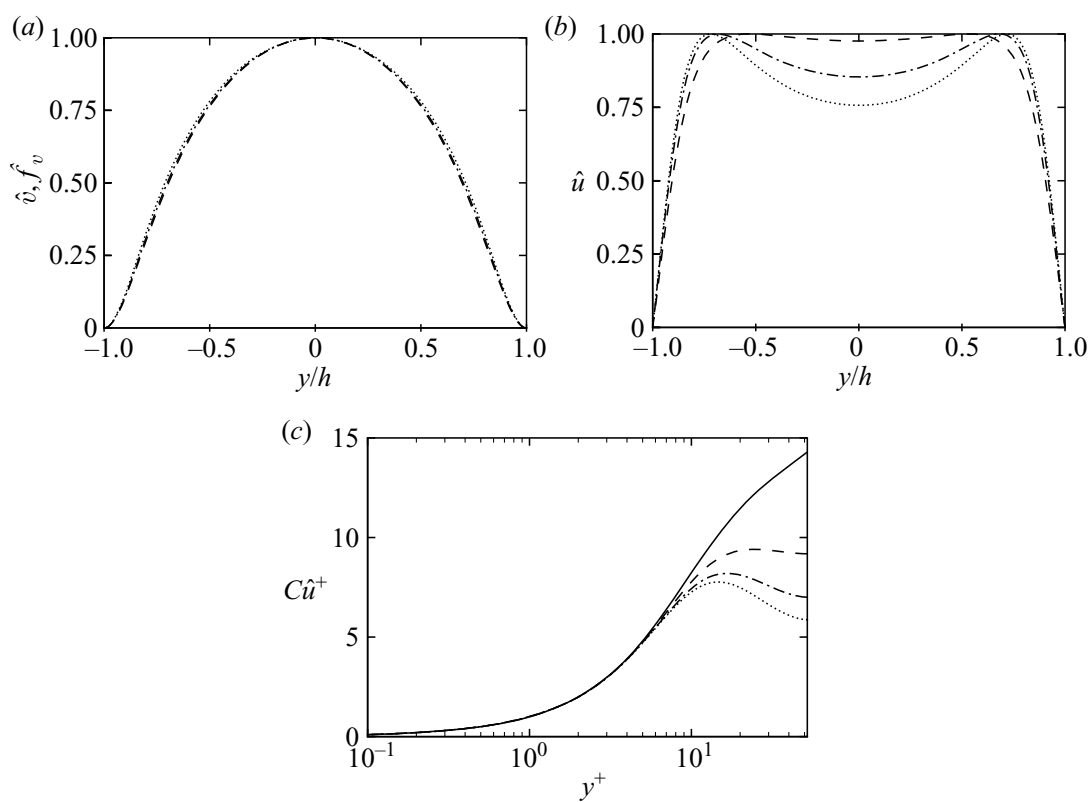

FIGURE 6. Wall-normal dependence of (a) the wall-normal Fourier component $\hat{v}(y / h), \hat{f}_{y}(y / h)$ corresponding to the optimal input fields (figure $5 a, c, e)$ and of $(b)$ the streamwise Fourier component $\hat{u}(y / h)$ corresponding to the optimal output velocity fields (figure $5 b, d, f$ ). (c) Same as in $(a)$ but in wall units: $C \hat{u}^{+}\left(y^{+}\right)$with the constant $C$ chosen so as to match the mean velocity field $U^{+}\left(y^{+}\right)$reported in the same figure $(-)$. In $(a)$ and $(b)$, the maximum values have been normalized to 1 to allow for comparison. Data from the initial value problem $(\cdots \cdots \ldots . .$.$) and from the stochastic (-\cdot-\cdot-)$ and harmonic $\left(---^{-}\right)$forcing problems.

perturbations has been modelled with the Orr-Sommerfeld-Squire system generalized to include the variable eddy viscosity following Reynolds \& Hussain (1972), del Álamo \& Jiménez (2006), Pujals et al. (2009) and Cossu et al. (2009). The main results are as follows:

(a) The computed turbulent mean flow at $R e=750$ is well converged for averaging times of the order of $700 h / U_{w}$ where it displays $R e_{\tau}=52$. The mean velocity profile and the associated turbulent shear stress show good agreement with previous results of Komminaho et al. (1996) and Kitoh et al. (2005).

(b) The turbulent mean flow is asymptotically stable (it has stable eigenvalues).

(c) The maximum temporal energy growth is small (less than 6.5 at this low Reynolds number) but the responses to the external forcing are larger. The energy amplification of the optimal deterministic forcing is two orders of magnitude larger than the maximum amplification of the variance induced by stochastic forcing.

(d) In the case of stochastic forcing with the wavenumbers inducing maximum variance, almost $85 \%$ of the variance is due to the first Karhunen-Loève mode.

(e) Only structures elongated in the streamwise direction are significantly amplified, and the most amplified ones are streamwise uniform $\left(\alpha=0, \lambda_{x}=\infty\right)$.

$(f)$ For streamwise uniform perturbations, the spanwise wavelength maximizing the temporal energy growth is $\lambda_{z}=4.4 h$, the one maximizing the amplification of the variance of stochastic forcing is $\lambda_{z}=5.2 \mathrm{~h}$ while the maximum amplification of harmonic forcing is obtained for the larger spanwise wavelength $\lambda_{z}=7.7 \mathrm{~h}$ and with steady forcing $\left(\omega_{f}=0\right)$. 
$(g)$ For all the three types of input-output problems, the optimal output velocity fields have a dominant streamwise component (streaks) while the input has dominant cross-stream components (streamwise vortices).

(h) The wall-normal profile of the wall-normal component of the three considered optimal inputs is essentially the same, even if the physical significance of each input is different from the others.

(i) The profiles of the optimal (output) large-scale streaks pertaining to the three considered problems are proportional to the turbulent mean profile up to buffer layer $\left(y^{+} \lesssim 8-10\right.$ for this very low Reynolds number).

These results are consistent with previous input-output analyses of laminar and turbulent wall-bounded flows. In particular: (i) the maximum growths $G_{\max }$ sustained by the turbulent mean flow are small compared to the ones sustained by the laminar flow at the same $R e$; the associated optimal $\lambda_{z}$ in the turbulent case is larger than the optimal value in the laminar case. (ii) Only streamwise elongated structures are amplified with the optimal output given by streaks and the optimal input by streamwise vortices indicating that in all three cases (initial value problem, stochastic and harmonic forcing) a 'mean' lift-up mechanism is responsible for the energy amplifications. (iii) The ordering of the optimal spanwise wavelengths (the largest optimal corresponding to harmonic forcing, the shortest to the initial value problem with the one corresponding to the stochastic forcing lying in between) is also consistent with previous findings in laminar flows (no result is currently available for any other turbulent flow). (iv) The proportionality of the optimal large-scale streak profiles to the mean velocity profile near the wall is consistent with previous findings for the initial value problem in the turbulent Poiseuille and boundary layer flows. We prove that this feature also extends to the responses to harmonic and stochastic forcing.

The scales of the optimal streamwise uniform structures having the largest response to initial conditions $\left(\lambda_{z}=4.4 h\right)$ and stochastic forcing $\left(\lambda_{z}=5.2 h\right)$ show good agreement with those of the most energetic coherent structures observed in the numerical simulations of Komminaho et al. (1996) $\left(\lambda_{z}=4.2 \mathrm{~h}\right.$ and $\lambda_{x}>30 \mathrm{~h}$ at $\left.R e=750\right)$, Tsukahara et al. (2006) $\left(\lambda_{z}=4.2-5 h\right.$ and $\lambda_{x} \approx 42-64 h$ at $R e=750$ and 2150) and in the experiments of Tillmark \& Alfredsson (1994), Tillmark (1995) $\left(\lambda_{z} \approx 4-5 h\right.$ and $\lambda_{x}>30 h$ at $\left.R e=3300\right)$ and Kitoh \& Umeki (2008) $\left(\lambda_{z}=4 h\right.$ and $\lambda_{x} \approx 40-60 h$ at $R e=3750$ ). Furthermore, Tsukahara et al. (2006) have found that at $R e=750$ the most energetic POD modes have scales $\lambda_{z}=4.2-5.1 h$ and $\lambda_{x} \approx 45 h$. These most energetic POD modes show a striking resemblance with the most energetic mode of the response to stochastic forcing reported in our figure $6(f)$; the position of the peak in the $u$ response, at $y^{+}=15-20$, is also similar. In all these cases, the observed coherent streaks were not artificially forced, so that it is not surprising that the relevant scales come from the response to stochastic forcing and to initial conditions if one assumes that the effect of the nonlinear terms and of the small-scale fluctuations can be treated either as a stochastic forcing or as random initial conditions.

From the viewpoint of flow control and sensitivity to boundary conditions, where the forcing is deterministic and correlated over all times, then, the relevant scales should come from the harmonic forcing analysis. In this case, the present results indicate that the optimal response is obtained with the spanwise spacing $\lambda_{z}=7.7 \mathrm{~h}$, larger than the one arising in the (unforced) observed large-scale coherent streak $\left(\lambda_{z}=4 h-5 h\right)$. Furthermore, the very large response to harmonic forcing indicates good controllability of the large-scale structures even at low Reynolds numbers. This extreme sensitivity of the turbulent Couette flow to steady forcing has also been reported by Kitoh et al. (2005) and confirmed by Kitoh \& Umeki (2008) who 
artificially forced the large-scale streaks using vortex generators. If such a strong sensitivity of large-scale streaks to deterministic forcing is confirmed, then one could imagine to use them to modify the mean flow properties with specific targets. This kind of approach has already proven successful in the laminar boundary layer where optimal laminar streaks have been used to stabilize Tollmien-Schlichting waves and delay transition (Cossu \& Brandt 2002; Fransson et al. 2006).

Our results confirm the strong relation between the optimally amplified streaks and the large-scale coherent streaks measured in experiments and in DNS already reported for the turbulent Poiseuille flow (del Álamo \& Jiménez 2006; Pujals et al. 2009). The reasons of this strong correlation might however be more complex than they appear. In fact, the optimal perturbation approach provides a measure of the maximum amplification of streaks with given streamwise and spanwise scales but this amplification is only part of more complicated processes leading to the 'refuelling' of the vortices that induce the streaks. Mechanisms like the secondary instability of the streaks (Waleffe 1995; Reddy et al. 1998) or the transient amplification of streak bending (Schoppa \& Hussain 2002) are primordial to generate streamwise non-uniform perturbations leading to the refuelling of the quasi-streamwise vortices in the models of self-sustained turbulent cycles. These additional processes select particular streamwise and spanwise scales in the flow. The self-sustained scales that are actually observed, are therefore selected by the different mechanisms embedded in the self-sustained process. The correspondence, observed in the turbulent Couette and Poiseuille flows, of the scales of the large-scale streaks in the actual turbulent flows with the scales most amplified by the mean lift-up probably means that the scale selection from the additional processes is weak. It is important to remind that this situation could be not general. For instance, Cossu et al. (2009) found that in the turbulent boundary layers the optimal scale $\left(\lambda_{z} \approx 8 \delta\right)$ selected by the initial value problem is larger than the scale $\left(\lambda_{z} \approx \delta\right)$ actually observed for the coherent large-scale streaks (e.g. Jiménez et al. 2007). The search for self-sustained cycles of the largescale coherent structures in turbulent wall-bounded flows and the investigation of the mechanisms selecting their scales are the subject of current intensive investigation.

Y. Hwang acknowledges funding from the French Ministry of Foreign Affairs through a Blaise Pascal Scholarship. Partial support of DGA the use of the channelflow code (see http://www.channelflow.org) are also gratefully acknowledged.

\section{REFERENCES}

Del Álamo, J. C. \& JimÉnez, J. 2003 Spectra of the very large anisotropic scales in turbulent channels. Phys. Fluids 15, L41.

Del Álamo, J. C. \& JimÉnez, J. 2006 Linear energy amplification in turbulent channels. J. Fluid Mech. 559, 205-213.

Andersson, P., Brandt, L., Bottaro, A. \& Henningson, D. 2001 On the breakdown of boundary layers streaks. J. Fluid Mech. 428, 29-60.

Bamieh, B. \& Dahleh, M. 2001 Energy amplification in channel flows with stochastic excitation. Phys. Fluids 13, 3258-3269.

Butler, K. M. \& Farrell, B. F. 1992 Three-dimensional optimal perturbations in viscous shear flow. Phys. Fluids A 4, 1637-1650.

Butler, K. M. \& FARrell, B. F. 1993 Optimal perturbations and streak spacing in wall-bounded turbulent shear flow. Phys. Fluids 5, 774-777.

Cossu, C. \& Brandt, L. 2002 Stabilization of Tollmien-Schlichting waves by finite amplitude optimal streaks in the Blasius boundary layer. Phys. Fluids 14, L57-L60. 
Cossu, C., Pujals, G. \& Depardon, S. 2009 Optimal transient growth and very large scale structures in turbulent boundary layers. J. Fluid Mech. 619, 79-94.

Ellingsen, T. \& Palm, E. 1975 Stability of linear flow. Phys. Fluids 18, 487-488.

Farrell, B. F. \& IOANnou, P. J. 1993a Optimal excitation of three-dimensional perturbations in viscous constant shear flow. Phys. Fluids 5, 1390-1400.

Farrell, B. F. \& IOANNOU, P. J. $1993 b$ Stochastic forcing of the linearized Navier-Stokes equation. Phys. Fluids A 5, 2600-2609.

Farrell, B. F. \& IoAnnou, P. J. 1996 Generalized stability theory. J. Atmos. Sci. 53, 2025-2053.

FARRell, B. F. \& IOANnOU, P. J. 1998 Perturbation structure and spectra in turbulent channel flow. Theor. Comput. Fluid Dyn. 11, 237-250.

Fontane, J., Brancher, P. \& Fabre, D. 2008 Stochastic forcing of the Lamb-Oseen vortex. J. Fluid Mech. 613, 233-254.

Fransson, J., Talamelli, A., Brandt, L. \& Cossu, C. 2006 Delaying transition to turbulence by a passive mechanism. Phys. Rev. Lett. 96, 064501.

Gibson, J. F., Halcrow, J. \& Cvitanovic, P. 2008 Visualizing the geometry of state space in plane Couette flow. J. Fluid Mech. 611, 107-130.

Gustavsson, L. H. 1991 Energy growth of three-dimensional disturbances in plane Poiseuille flow. J. Fluid Mech. 224, 241-260.

Hamilton, J. M., Kim, J. \& WalefFe, F. 1995 Regeneration mechanisms of near-wall turbulence structures. J. Fluid Mech. 287, 317-348.

Ho, C. M. \& Huerre, P. 1984 Perturbed shear layers. Annu. Rev. Fluid Mech. 16, 365-424.

Hutchins, N. \& Marusic, I. $2007 a$ Evidence of very long meandering features in the logarithmic region of turbulent boundary layers. J. Fluid Mech. 579, 1-28.

Hutchins, N. \& Marusic, I. $2007 b$ Large-scale influences in near-wall turbulence. Phil. Trans. R. Soc. A 365, 647-664.

JANG, P. S., Benney, D. J. \& GRAN, D. L. 1986 On the origin of streamwise vortices in a turbulent boundary layer. J. Fluid Mech. 169, 109-123.

JimÉnEZ, J. 2007 Recent developments on wall-bounded turbulence. Rev. R. Acad. Cien. Ser. A Mat. 101, 187-203.

JimÉnez, J. \& Moin, P. 1991 The minimal flow unit in near-wall turbulence. J. Fluid Mech. 225, 213-240.

Jovanović, M. R. \& Bamieh, B. 2005 Componentwise energy amplification in channel flow. J. Fluid Mech. 543, 145-83.

Kim, K. C. \& Adrian, R. 1999 Very large-scale motion in the outer layer. Phys. Fluids 11 (2), 417-422.

Kitoh, O., Nakabayashi, K. \& Nishimura, F. 2005 Experimental study on mean velocity and turbulence characteristics of plane Couette flow: low-Reynolds-number effects and large longitudinal vortical structures. J. Fluid Mech. 539, 199.

KiтoH, O. \& UMEKI, M. 2008 Experimental study on large-scale streak structure in the core region of turbulent plane Couette flow. Phys. Fluids 20, 025107.

Kline, S. J., Reynolds, W. C., Schraub, F. A. \& Runstadler, P. W. 1967 The structure of turbulent boundary layers. J. Fluid Mech. 30, 741-773.

Komminaho, J., Lundbladh, A. \& Johansson, A. V. 1996 Very large structures in plane turbulent Couette flow. J. Fluid Mech. 320, 259-285.

LANDAHL, M. T. 1980 A note on an algebraic instability of inviscid parallel shear flows. J. Fluid Mech. 98, 243-251.

LANDAHL, M. T. 1990 On sublayer streaks. J. Fluid Mech. 212, 593-614.

LEE, M. J. \& KIM, J. 1991 The structure of turbulence in a simulated plane Couette flow. In Eighth Symp. on Turbulent Shear Flow, pp. 5.3.1-5.3.6. Technical University of Munich, Munich, Germany.

Moffatt, H. K. 1967 The interaction of turbulence with strong wind shear. In Proceedings of URSI-IUGG Colloquium on Atomspheric Turbulence and Radio Wave Propagation (ed. A. M. Yaglom \& V. I. Tatarsky), pp. 139-154. Nauka.

Morn, P. \& Kim, J. 1982 Numerical investigation of turbulent channel flow. J. Fluid Mech. 118, 341-377. 
Pujals, G., García-Villalba, M., Cossu, C. \& Depardon, S. 2009 A note on optimal transient growth in turbulent channel flows. Phys. Fluids 21, 015109.

Reddy, S. C. \& Henningson, D. S. 1993 Energy growth in viscous channel flows. J. Fluid Mech. 252, 209-238.

Reddy, S. C., Schmid, P. J., Baggett, J. S. \& Henningson, D. S. 1998 On the stability of streamwise streaks and transition thresholds in plane channel flows. J. Fluid Mech. 365, 269-303.

ReYnolds, W. C. \& Hussain, A. K. M. F. 1972 The mechanics of an organized wave in turbulent shear flow. Part 3. Theoretical models and comparisons with experiments. J. Fluid Mech. 54 (02), 263-288.

Schmid, P. J. 2007 Nonmodal stability theory. Annu. Rev. Fluid Mech. 39, 129-162.

Schmid, P. J. \& Henningson, D. S. 1994 Optimal energy density growth in Hagen-Poiseuille flow. J. Fluid Mech. 277, 197-225.

Schmid, P. J. \& Henningson, D. S. 2001 Stability and Transition in Shear Flows. Springer.

Schoppa, W. \& Hussain, F. 2002 Coherent structure generation in near-wall turbulence. J. Fluid Mech. 453, 57-108.

Smith, J. R. \& Metzler, S. P. 1983 The characteristics of low-speed streaks in the near-wall region of a turbulent boundary layer. J. Fluid Mech. 129, 27-54.

Tillmark, N. 1995 Experiments on transition and turbulence in plane Couette flow. PhD thesis, Department of Mechanics, Royal Institute of Technology (KTH), Stockholm.

Tillmark, N. \& Alfredsson, H. 1994 Structures in turbulent plane Couette flow obtained from correlation measurements. In Advances in Turbulences $V$ (ed. R. Benzi), pp. 502-507. Kluwer.

Tomkins, C. D. \& AdRian, R. J. 2003 Spanwise structure and scale growth in turbulent boundary layers. J. Fluid Mech. 490, 37-74.

Tomkins, C. D. \& Adrian, R. J. 2005 Energetic spanwise modes in the logarithmic layer of a turbulent boundary layer. J. Fluid Mech. 545, 141-162.

Trefethen, L. N., Trefethen, A. E., Reddy, S. C. \& Driscoll, T. A. 1993 A new direction in hydrodynamic stability: beyond eigenvalues. Science 261, 578-584.

Tsukahara, T., Iwamoto, K. \& Kawamura, H. 2007 POD analysis of large-scale structures through DNS of turbulence Couette flow. In Advances in Turbulence XI (ed. J. M. L. M. Palma \& A. Silva Lopes) pp. 245-247. Springer.

Tsukahara, T., Kawamura, H. \& ShingaI, K. 2006 DNS of turbulent Couette flow with emphasis on the large-scale structure in the core region. J. Turbul. 7, 19.

WALEFFE, F. 1995 Hydrodynamic stability and turbulence: beyond transients to a self-sustaining process. Stud. Appl. Math. 95, 319-343.

Weideman, J. A. C. \& Reddy, S. C. 2000 A MATLAB differentiation matrix suite. ACM Trans. Math. Softw. 26, 465-519.

Zhou, K., Doyle, J. C. \& Glover, K. 1996 Robust and Optimal Control. Prentice Hall. 